\title{
ConheceDOR: the development of a board game for modern pain education for patients with musculoskeletal pain
}

\author{
ConheceDOR: desenvolvimento de um jogo de tabuleiro para educação moderna em dor para \\ pessoas com dor musculoesquelética
}

Juliana Carvalho de Paiva Valentim¹, Ney Armando Meziat-Filho², Leandro Calazans Nogueira², Felipe J. Jandre Reis ${ }^{3}$

DOI 10.5935/2595-0118.20190030

\begin{abstract}
BACKGROUND AND OBJECTIVES: Chronic pain is one of the main challenges for health systems. Pain education and self-motivated strategies have great potential in the treatment of people with chronic pain, especially by modifying beliefs and behavior. The development of board games for educational purposes can contribute to the learning of pain concepts and behavioral strategies. The objective of this study was to develop a board game (ConheceDor) to be used as an intervention tool for pain education.
\end{abstract}

CONTENTS: The systematic review for the development of the game ConheceDor, considered the following search strategy: "chronic pain", "musculoskeletal pain", "health education", "patient education", "neuroscience education”, "pain education", "therapeutic neuroscience education". The primary outcomes considered were pain intensity and disability. Fifteen studies were included, with a total of 1,486 participants. Six studies reported reduction on pain of at least $10 \%$, and two studies reported an improvement of at least $30 \%$ on disability. For the development of the game, we elaborated the layout of the board, the rules and other components (dice, cards, and pins). The cards of the game included the contents commonly used in the randomized controlled trials: negative thoughts, pain neurophysiology, stress management, and relaxation, coping and exercises.

CONCLUSION: The development of the present board game was based on the critical appraisal of the content of education-

Juliana Carvalho de Paiva Valentim - (Dhttps://orcid.org/0000-0001-9522-8523;

Ney Armando Meziat-Filho - (1) https://orcid.org/0000-0003-2794-7299;

Leandro Calazans Nogueira - Dhttps://orcid.org/0000-0002-0177-9816;

Felipe J. Jandre Reis - (Dhttps://orcid.org/0000-0002-9471-1174.

1. Instituto Federal de Educaçấo, Ciência e Tecnologia do Rio de Janeiro, Rio de Janeiro, RJ, Brasil. 2. Centro Universitário Augusto Motta, Programa de Pós-Graduação em Ciências da Reabilitação, Rio de Janeiro, RJ, Brasil.

3. Universidade Federal do Rio de Janeiro, Instituto Federal de Educação, Ciência e Tecnologia do Rio de Janeiro, Rio de Janeiro, RJ, Brasil.

Submitted on July 09, 2018.

Accepted for publication on December 10, 2018.

Conflict of interests: none - Sponsoring sources: none.

Correspondence to:

Instituto Federal do Rio de Janeiro, Campus Realengo

Rua Carlos Wenceslau, 343 - Realengo

21715-000 Rio de Janeiro, RJ, Brasil.

E-mail: felipe.reis@ifrj.edu.br

(C) Sociedade Brasileira para o Estudo da Dor al strategies present in the literature. The board game can be a potent resource to be applied in clinical practice in people with musculoskeletal pain.

Keywords: Chronic pain, Experimental games, Health education, Neuroscience-based education.

\section{RESUMO}

JUSTIFICATIVA E OBJETIVOS: A dor crônica é um dos principais desafios para os sistemas de saúde. As estratégias comportamentais e a educaçáo em dor apresentam grande potencial no tratamento de pessoas com dor crônica, especialmente pela modificação de crenças e do comportamento. Os jogos de tabuleiro podem ser uma estratégia educativa que contribui para a aprendizagem dos conceitos sobre dor e estratégias comportamentais. O objetivo deste estudo foi desenvolver um jogo de tabuleiro (ConheceDor) como ferramenta de intervenção para educação em dor.

CONTEÚDO: A revisão sistemática para o desenvolvimento do jogo ConheceDor, considerou a estratégia de busca com os descritores "chronic pain", "musculoskeletal pain", "health education", "patient education”, "neuroscience education”, "pain education”, "therapeutic neuroscience education". Os desfechos primários considerados foram a intensidade da dor e a incapacidade. Foram incluídos 15 estudos com um total de 1.486 participantes. Seis estudos apresentaram redução da dor de pelo menos $10 \%$ e dois estudos atingiram uma melhora de pelo menos 30\% na incapacidade. Para o desenvolvimento do jogo foram elaborados o layout do tabuleiro, as regras e os demais componentes (dados, cartas, pinos). As cartas do ConheceDor incluíram os temas mais utilizados nos estudos identificados que foram: pensamentos negativos, neurofisiologia da dor, manuseio do estresse e relaxamento, enfrentamento e exercícios físicos.

CONCLUSÃO: A criação de um jogo de tabuleiro considerou uma análise crítica da literatura dos conteúdos das estratégias educativas presentes nos ensaios clínicos. O desenvolvimento dessa intervenção pode ser um recurso para ser aplicado na prática clínica em pessoas com dor musculoesquelética.

Descritores: Dor crônica, Educação com base em neurociência, Educação em saúde, Jogos experimentais.

\section{INTRODUCTION}

The health conditions that are characterized by the presence of pain can lead to disability and high costs to the individual and 
society. Chronic musculoskeletal pain, especially cervical and low back pain are among the main health conditions associated with disability ${ }^{1}$. Nowadays, cognitive-emotional and behavioral factors, in addition to the physical factors, are considered as contributing to the disability observed in people with chronic pain $^{2-4}$.

Pain may be associated with the presence of emotional changes such as catastrophic thoughts, anxiety, fear, kinesiophobia, maladaptive behaviors, and depression ${ }^{5-8}$. The literature highlights pain education (PE) and behavioral interventions to intervene in this components ${ }^{9,10}$. Recently, a systematic review evaluated the efficacy of pain neuroscience education in patients with chronic musculoskeletal pain and identified that the intervention contributed to the reduction of pain, disability, and catastrophization $^{11}$. Behavioral and cognitive interventions help in the deconstruction of negative thinking patterns, beliefs, emotional states, and maladaptive behaviors, with the main objectives of reducing symptom-related distress, improving functionality, assisting in changing adaptive patterns, and teaching techniques for the self-management of pain ${ }^{12}$.

Educational games can contribute to the learning process since they favor the interaction of the participants and the assimilation of concepts in a playful way ${ }^{13,14}$. Considering that the development of individuals is related to the learning process acquired through the socio-cultural interaction ${ }^{13}$, the use of games as a teaching-learning proposal allows the content to be presented and the concepts constructed during the course of the game ${ }^{14}$. Educational games can be relevant tools used in the PE process to teach and learn the contents as well as for the modification of behaviors through the construction of knowledge.

The objective of this study was to develop a board game (Knowing Pain, in Portuguese ConheceDor) to serve as a tool to promote the knowledge about the pain concepts and the motivational strategies for people with chronic musculoskeletal pain.

\section{CONTENTS}

A systematic review of the literature of intervention studies using PE or behavioral strategies was carried out to develop the board game. The protocol defined for this study followed the recommendations of the Preferred Reporting Items for Systematic Reviews and Meta-Analyzes (PRISMA). The question of the review was: "what are the contents of education and pain relief interventions used in randomized clinical trials that have promoted benefits for pain and disability outcomes in people with chronic musculoskeletal pain"? These contents, identified exclusively in clinical trials, were used to elaborate the domains of the board game ConheceDor.

\section{Inclusion and exclusion criteria}

The studies considered were randomized controlled trials that investigated the effects of PE based on neuroscience or behavioral, self-management or motivational strategies on pain and disability. The studies should include participants over 18 years of age and with musculoskeletal pain lasting longer than 12 weeks. Interventions could be done in person or at distance (online, telehealth, among others), individually or in a group. In case there has been an intervention with contact with the health professional and the other group having received the intervention at a distance, the in-person intervention was used. The studies should be available for access, and there was no restriction on the language of publication provided that the translation was available. We excluded studies that used as main intervention education based on only biomedical concepts, as well as those that provided an orientation of posture or only orientations based on purely biomechanical concepts. The exclusion of this type of study was because these concepts contribute to the increase of catastrophization, anxiety, and fear related to pain ${ }^{11}$. The control groups could be from usual treatment, waiting list or other educational strategies. Also, PE or behavioral strategies could be associated with other interventions such as exercises, for example. The primary outcomes considered for the study were pain intensity and disability. The secondary outcomes included changes in catastrophizing, kinesiophobia, anxiety, depression, improvement perception, patient satisfaction, and return to work. In addition to these criteria, a minimum score of six on the PEDro scale was adopted for methodological quality.

\section{Search strategy for the identification of the studies}

A search was performed on the Pubmed, PEDro, Scopus, and Web of Science databases in February 2018. The search strategy utilized the following descriptors: "chronic pain" OR "musculoskeletal pain" AND "health education" OR "patient education" OR "neuroscience education" OR "pain education" OR "therapeutic neuroscience education" (Annex 1). The search terms were adapted for use with other bibliographic databases in combination with specific database filters for controlled trials, when available.

\section{Selection of studies and data extraction}

After the search, the results were imported to the EndNote Web. Considering the eligibility criteria, two researchers independently selected the potentially eligible articles based on the title, abstract and full text. There was no blinding for the journal or authors. The data extraction was performed, and the divergences were resolved by consensus. In the absence of consensus, a third evaluator could be convened. The data extracted included the author (year), clinical condition, population, intervention group, the control group, follow-up, outcomes and content of interventions.

\section{Data analysis}

In order to identify the contents used in the educational interventions of the clinical trials included in the present study, the data analysis was performed descriptively. The intragroup difference between baseline and post-intervention measures was considered to attest the modification of the interventions on pain and disability outcomes. The follow-up time was grouped in short (up to 3 months), medium (3 to 6 months) and long (over 6 months), and the largest follow-ups were considered for grouping. The change in outcomes was presented as a percentage. The 
reduction of at least $10 \%$ in pain intensity and an improvement of at least $30 \%$ in disability was considered a significant change. After identifying the studies that reached these values of change, the contents of the interventions were grouped by similarity and presented by their absolute frequencies.

The overall quality of the evidence was evaluated using the PEDro scale which has 11 items and a total score of 10 according to the following criteria: eligibility criteria; random allocation; secret allocation; comparability at the baseline; participants; blind therapist surveyors; suitable follow-up; intention-to-treat analysis; comparison between groups; accuracy and variability. The highest score on the PEDro scale indicated better methodological quality.

\section{Board game development}

After the identification of the studies, the development of the ConheceDor board game began. For this stage, two researchers identified the contents of the interventions used in the studies included in the systematic review and then developed the main elements to visually compose the board that included the layout, the number, and content of the houses and cards, the rules and other components (dice and pins). For the development of the charts of the board domains, the content addressed in at least $50 \%$ of the studies was selected.

\section{Description of the studies}

The initial search identified a total of 2,907 studies. Of these, 1,945 duplicate studies were excluded. The screening of titles and abstracts identified 44 potential articles and, after a detailed analysis of the full text of the studies, the final sample for analysis was composed of 15 studies (Figure 1). The main reasons for the exclusion of the articles were: not being a clinical trial, not describing how the educational proposal was performed and presenting a score lower than six on the PEDro scale. The studies that did not meet the inclusion criteria were excluded, that is, access not available $(n=3)$; educational content not described $(n=2)$ or education combined with other intervention $(n=1)$; no evaluation of the outcomes considered for the inclusion $(n=1)$; addressing patients with acute pain or cancer-related pain $(n=2)$; using posture-orientation based content $(n=2)$ and with a PEDro score $<6(n=18)$.

\section{Characteristics of the articles included}

Fifteen studies were included, totaling 1,486 people with chronic musculoskeletal pain. Five studies with people with chronic low back pain ${ }^{15-19}$, four studies with musculoskeletal pain of different origins ${ }^{20-23}$, two studies with participants with chronic neck pain ${ }^{24,25}$, one study with patients with chronic fatigue syndrome ${ }^{26}$, one study with fibromyalgia ${ }^{27}$, one study with participants with spinal pain or upper back pain ${ }^{28}$, one study with patients with chronic knee pain ${ }^{29}$.

Among the interventions, seven studies (46.7\%) used neuroscience-based PE $15,17,18,22,25,26,29$, five studies (33.4\%) used PE and behavioral strategies ${ }^{20,21,23,24,27}$, one study $(6.7 \%)$ used only behavioral strategies ${ }^{19}$, one study $(6.7 \%)$ used general health guidelines ${ }^{28}$ and one study (6.7\%) associated neuroscience-based PE with hypnosis ${ }^{16}$. Table 1 presents the characteristics of the studies included in the systematic review.

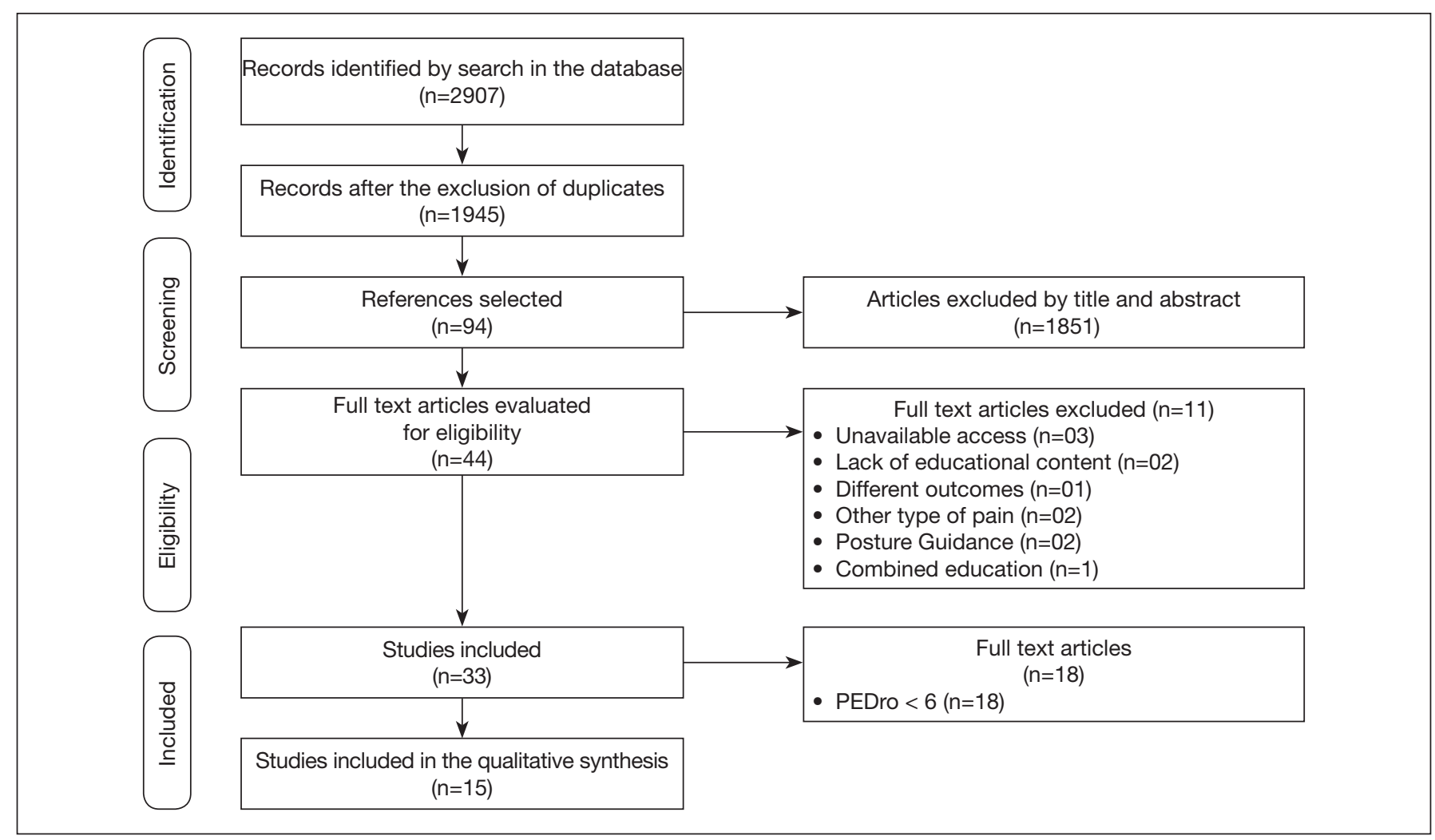

Figure 1. The selection process of the studies for inclusion in the systematic review 
Table 1. Summary of the studies included $(n=15)$

\begin{tabular}{|c|c|c|c|c|c|c|}
\hline Authors & Clinical condition & Population & Intervention group & Control group & Follow-up & Outcomes \\
\hline $\begin{array}{l}\text { Andersen et } \\
\text { al. }{ }^{28}\end{array}$ & $\begin{array}{l}\text { Pain in the spine } \\
\text { or upper back }\end{array}$ & $\begin{array}{l}\mathrm{n}=141 \\
\mathrm{~F}=78 \\
\text { Age }=45.2 \pm 0.5 \\
\text { years }\end{array}$ & $\begin{array}{l}\text { Personalized physical } \\
\text { activity group }=1.5 \mathrm{~h} \\
\text { of general health gui- } \\
\text { delines }+ \text { aerobic and } \\
\text { strength exercises for } \\
50 \text { min }(n=47) \text {. } \\
\text { Group of sel- } \\
\text { f-management }=1.5 \mathrm{~h} \text { of } \\
\text { general health guideli- } \\
\text { nes }+ \text { weekly workshop } \\
\text { of } 2.5 \mathrm{~h} \text { for } 6 \text { weeks in } \\
\text { groups of } 12 \text { to } 18 \text { peo- } \\
\text { ple }(n=47) \text {. }\end{array}$ & $\begin{array}{l}\text { Reference treatment } \\
\text { group }=1.5 \mathrm{~h} \text { of ge- } \\
\text { neral health guideli- } \\
\text { nes }(n=47) \text {. }\end{array}$ & 3 months & $\begin{array}{l}\text { Intensity of pain } \\
\text { with VAS (zero to } \\
100 \mathrm{~mm} \text { ); } \\
\text { Ability to work (VAS } \\
\text { zero to 100mm); } \\
\text { TSK. }\end{array}$ \\
\hline $\begin{array}{l}\text { Bennell et } \\
\text { al. }{ }^{29}\end{array}$ & $\begin{array}{l}\text { Chronic knee } \\
\text { pain }\end{array}$ & $\begin{array}{l}\mathrm{n}=148 \\
\mathrm{~F}=83 \\
\text { Age }=61.1 \pm 7.5 \\
\text { years }\end{array}$ & $\begin{array}{l}\text { Three internet interven- } \\
\text { tions: educational ma- } \\
\text { terial on the exercises } \\
+ \text { educational material } \\
\text { on coping }+7 \text { consul- } \\
\text { tations via Skype with } \\
\text { a physiotherapist for } \\
\text { exercise prescription } \\
(n=74) \text {. }\end{array}$ & $\begin{array}{l}\text { Two interventions } \\
\text { via the internet: edu- } \\
\text { cational material on } \\
\text { the exercises + edu- } \\
\text { cational material on } \\
\text { coping }(n=74) \text {. }\end{array}$ & $\begin{array}{l}3 \text { and } 9 \text { mon- } \\
\text { ths }\end{array}$ & $\begin{array}{l}\text { Mean pain during } \\
\text { gait in the previous } \\
\text { week (zero to 10); } \\
\text { WOMAC and PSC. }\end{array}$ \\
\hline $\begin{array}{l}\text { Brage et } \\
\text { al. }^{24}\end{array}$ & $\begin{array}{l}\text { Chronic neck } \\
\text { pain }\end{array}$ & $\begin{array}{l}\mathrm{n}=20 \\
\mathrm{~F}=20 \\
\text { Age }=41.4 \pm 12.2 \\
\text { years }\end{array}$ & $\begin{array}{l}4 \text { sessions of } 1.5 \mathrm{~h} \text { on } \\
\text { education }+8 \text { sessions } \\
\text { of } 30 \mathrm{~min} \text { with speci- } \\
\text { fic exercises (shoulder } \\
\text { girdle and shoulder, } \\
\text { balance and aerobic } \\
\text { training) }(n=10) \text {. }\end{array}$ & $\begin{array}{l}4 \text { sessions of } 1.5 \mathrm{~h} \text {, } \\
\text { covering topics on } \\
\text { mechanisms, ac- } \\
\text { ceptance, coping } \\
\text { strategies and defi- } \\
\text { nition of pain goals } \\
\text { based on the con- } \\
\text { cepts of pain mana- } \\
\text { gement and cogniti- } \\
\text { ve-behavioral thera- } \\
\text { py }(n=10) \text {. }\end{array}$ & $\begin{array}{l}4 \text { and } 12 \\
\text { months }\end{array}$ & $\begin{array}{l}\text { Pain (NRS from zero } \\
\text { to 10); } \\
\text { Neck-related disabi- } \\
\text { lity (NDI); } \\
\text { Perceived Global Ef- } \\
\text { fect (GPE). }\end{array}$ \\
\hline $\begin{array}{l}\text { Carmody et } \\
\text { al. }{ }^{23}\end{array}$ & $\begin{array}{l}\text { Chronic muscu- } \\
\text { loskeletal pain } \\
\text { (low back and } \\
\text { neck pain, with } \\
\text { and without radi- } \\
\text { culopathy, arth- } \\
\text { ritis) }\end{array}$ & $\begin{array}{l}\mathrm{n}=101 \\
\mathrm{~F}=3 ; \\
\text { Age }=67.5 \pm 9.5 \\
\text { years }\end{array}$ & $\begin{array}{l}\text { Cognitive behavioral } \\
\text { therapy by telephone } \\
\text { (12 weeks) }(n=50)\end{array}$ & $\begin{array}{l}\text { Telephone } \quad \text { edu- } \\
\text { cation (12 } \text { weeks }) \\
(n=51)\end{array}$ & $\begin{array}{l}2,5,8 \text { and } \\
12 \text { months }\end{array}$ & $\begin{array}{l}\text { Health-related qual- } \\
\text { ity of life (SF-12v2); } \\
\text { BDI; PBCL; CSQR; } \\
\text { Intensity of pain } \\
\text { (pain journal for two } \\
\text { weeks); } \\
\text { PSC. }\end{array}$ \\
\hline $\begin{array}{l}\text { Chiauzzi et } \\
\text { al. }^{15}\end{array}$ & $\begin{array}{l}\text { Chronic low back } \\
\text { pain }\end{array}$ & $\begin{array}{l}\mathrm{n}=199 \\
\mathrm{~F}=134 \\
\text { Age }=46.1 \pm 11.9 \\
\text { years }\end{array}$ & $\begin{array}{l}\text { Online education } \\
\text { for low back pain } \\
\text { (painACTION-Back Pain) } \\
\text { (painACTION-Back } \\
\text { Pain) } n=104 \text { ) }\end{array}$ & $\begin{array}{l}\text { Low back pain guide } \\
\text { that should be read } \\
\text { in } 4 \text { weeks }(n=105)\end{array}$ & $\begin{array}{l}1,3 \text { and } 6 \\
\text { months }\end{array}$ & $\begin{array}{l}\text { BPI, ODQ, DASS, } \\
\text { PGIC, CPCI, PSC, } \\
\text { PSEQ FABQ. }\end{array}$ \\
\hline $\begin{array}{l}\text { Gallagher, } \\
\text { McAuley } \\
\text { and } \\
\text { Moseley }^{22}\end{array}$ & $\begin{array}{l}\text { Chronic muscu- } \\
\text { loskeletal pain }\end{array}$ & $\begin{array}{l}\mathrm{n}=79 \\
\mathrm{~F}=48 \\
\mathrm{Age}=43.5 \pm 11 \text { years }\end{array}$ & $\begin{array}{l}\text { Booklet on pain educa- } \\
\text { tion through metaphors } \\
(n=40) \text {. }\end{array}$ & $\begin{array}{l}\text { Information booklet } \\
\text { on pain }(n=39) \text {. }\end{array}$ & 3 months & $\begin{array}{l}\text { PBQ, PSC, NRS } \\
0-10, \text { PSFS. }\end{array}$ \\
\hline $\begin{array}{l}\text { Lefort et } \\
\text { al. } 20\end{array}$ & $\begin{array}{l}\text { Chronic muscu- } \\
\text { loskeletal pain }\end{array}$ & $\begin{array}{l}\mathrm{n}=110 \\
\mathrm{~F}=82 \\
\text { Age }=39.5 \text { years }\end{array}$ & $\begin{array}{l}\text { Psychoeducation pro- } \\
\text { gram ( } 2 \text { hours per week } \\
\text { for } 6 \text { weeks) ( } n=57)\end{array}$ & Waiting list $(n=53)$ & 6 weeks & $\begin{array}{l}\text { SF-36, PRI, SF-M- } \\
\text { PQ, SF-BDI; SOPA- } \\
\text {-D, VAS=100mm. }\end{array}$ \\
\hline $\begin{array}{l}\text { Meeus et } \\
\text { al. }^{26}\end{array}$ & $\begin{array}{l}\text { Chronic fatigue } \\
\text { syndrome }\end{array}$ & $\begin{array}{l}\mathrm{n}=48 \\
\mathrm{~F}=40 \\
\text { Age }=40.3 \pm 10.4 \\
\text { years }\end{array}$ & $\begin{array}{l}\text { Neuroscience-based } \\
\text { pain education }(n=24)\end{array}$ & $\begin{array}{l}\text { Information on self- } \\
\text {-management see- } \\
\text { king the balance } \\
\text { between activity and } \\
\text { rest to avoid exacer- } \\
\text { bations and esta- } \\
\text { blish realistic goals } \\
\text { to increase activity } \\
(n=24) \text {. }\end{array}$ & $\begin{array}{l}\text { Post-treat- } \\
\text { ment }\end{array}$ & $\begin{array}{l}\text { NPT, PCS, TSK, } \\
\text { PCI. }\end{array}$ \\
\hline
\end{tabular}


Table 1. Summary of the studies included $(n=15)$

\begin{tabular}{|c|c|c|c|c|c|c|}
\hline Authors & Clinical condition & Population & Intervention group & Control group & Follow-up & Outcomes \\
\hline $\begin{array}{l}\text { Moseley, } \\
\text { Nicholas } \\
\text { and } \\
\text { Hodges }^{18}\end{array}$ & $\begin{array}{l}\text { Chronic low back } \\
\text { pain }\end{array}$ & $\begin{array}{l}\mathrm{n}=58 \\
\mathrm{~F}=33 \\
\text { Age }=43.58 \text { years }\end{array}$ & $\begin{array}{l}\text { Neuroscience-based } \\
\text { pain education }(n=31)\end{array}$ & $\begin{array}{l}\text { Education on the } \\
\text { anatomy of the spi- } \\
\text { ne }(n=27)\end{array}$ & $\begin{array}{l}\text { Post-treat- } \\
\text { ment }\end{array}$ & $\begin{array}{l}\text { RMDQ, SOPA-R, } \\
\text { PSC. }\end{array}$ \\
\hline $\begin{array}{l}\text { Nicholas et } \\
\text { al. }{ }^{19}\end{array}$ & Chronic pain & $\begin{array}{l}\mathrm{n}=141 \\
\mathrm{~F}=96 \\
\text { Age }=73.9 \pm 6.5 \\
\text { years }\end{array}$ & $\begin{array}{l}\text { Self-management of } \\
\text { pain ( } 8 \text { sessions of } 2 h \\
\text { for } 4 \text { weeks) }+ \text { encou- } \\
\text { ragement to practi- } \\
\text { ce exercises at home } \\
(n=49) \\
\text { Self-management of } \\
\text { pain ( } 8 \text { sessions of } 2 h \\
\text { for } 4 \text { weeks) }(n=53)\end{array}$ & Waiting list $(n=39)$ & $\begin{array}{l}1,6 \text { and } 12 \\
\text { months }\end{array}$ & $\begin{array}{l}\text { NRS, mRMDQ; } \\
\text { DASS-21, TSK, } \\
\text { PSEQ, PRSS. }\end{array}$ \\
\hline Ris et al. ${ }^{25}$ & $\begin{array}{l}\text { Chronic neck } \\
\text { pain }\end{array}$ & $\begin{array}{l}\mathrm{n}=200 \\
\mathrm{~F}=149 \\
\text { Age }=45.1 \text { years }\end{array}$ & $\begin{array}{l}\text { Pain education with a } \\
\text { focus on the unders- } \\
\text { tanding/acceptance } \\
\text { of pain and goals set- } \\
\text { ting ( } 4 \text { sessions ( } 1.5 \mathrm{~h} \\
\text { each, once a month) } \\
+8 \text { sessions of exer- } \\
\text { cises (neck muscles, } \\
\text { balance, oculomotor } \\
\text { training, shoulder girdle } \\
(\mathrm{n}=101)\end{array}$ & $\begin{array}{l}\text { Pain Education } \\
\text { with a focus in the } \\
\text { understanding/ac- } \\
\text { ceptance of pain } \\
\text { and goals setting } \\
\text { ( } 4 \text { sessions (1.5h } \\
\text { each, once a month) } \\
(n=99)\end{array}$ & 4 months & $\begin{array}{l}\text { SF-36, NDI, BDI-II, } \\
\text { TSK. }\end{array}$ \\
\hline $\begin{array}{l}\text { Rizzo et al. } \\
{ }_{16}\end{array}$ & $\begin{array}{l}\text { Chronic low back } \\
\text { pain }\end{array}$ & $\begin{array}{l}\mathrm{n}=100 \\
\mathrm{~F}=80 \\
\text { Age }=50 \pm 13.5 \text { years }\end{array}$ & $\begin{array}{l}\text { Neuroscience-based } \\
\text { pain education }(4 \\
\text { sessions; } 2 \text { times/week) } \\
+ \text { hypnosis ( } 2 \mathrm{~h} \text { of self- } \\
\text { hypnosis in } 2 \text { weeks } \\
+ \text { book with hypnosis } \\
\text { suggestions) }(n=50)\end{array}$ & $\begin{array}{l}\text { Neuroscience-ba- } \\
\text { sed pain education } \\
(4 \text { sessions; } 2 \text { times/ } \\
\text { week) }(n=49)\end{array}$ & 3 months & $\begin{array}{l}\text { NRS, RMDQ, PSC, } \\
\text { GPE, PSFS. }\end{array}$ \\
\hline $\begin{array}{l}\text { Ryan et } \\
\text { al. }{ }^{17}\end{array}$ & $\begin{array}{l}\text { Chronic low back } \\
\text { pain }\end{array}$ & $\begin{array}{l}\mathrm{n}=38 \\
\mathrm{~F}=25 \\
\text { Age }=45.3 \pm 10.7 \\
\text { years }\end{array}$ & $\begin{array}{l}\text { Neuroscience-based } \\
\text { pain education }+6 \\
\text { sessions of exercises } \\
\text { for } 8 \text { weeks (10min } \\
\text { warm-up, 20-30min } \\
\text { aerobic phase, and } \\
10-15 \text { min slowdown) } \\
(n=20) \text {. }\end{array}$ & $\begin{array}{l}\text { Neuroscience-ba- } \\
\text { sed pain education } \\
(2.5 \mathrm{~h} \text { reformulation } \\
\text { of beliefs and attitu- } \\
\text { des regarding pain) } \\
(n=18)\end{array}$ & 3 months & $\begin{array}{l}\text { NRS, } \\
\text { PSEQ. }\end{array}$ \\
\hline $\begin{array}{l}\text { Thorn et } \\
\text { al. }{ }^{21}\end{array}$ & $\begin{array}{l}\text { Chronic muscu- } \\
\text { loskeletal pain }\end{array}$ & $\begin{array}{l}\mathrm{n}=73 \\
\mathrm{~F}=65 \\
\text { Age }=52.8 \pm 13.1 \\
\text { years }\end{array}$ & $\begin{array}{l}\text { Cognitive-Behavioral } \\
\text { Therapy + homework } \\
(1.5 \mathrm{~h}, \text { once/week, } 10 \\
\text { weeks) }(\mathrm{n}=49)\end{array}$ & $\begin{array}{l}\text { Education on pain } \\
\text { neurophysiology } \\
\text { ( } 1.5 \mathrm{~h} \text {, once/week, } \\
10 \text { weeks) }(n=34)\end{array}$ & 6 months & $\begin{array}{l}\text { BPI, RMDS, PSC, } \\
\text { CES-D, QOLS. }\end{array}$ \\
\hline $\begin{array}{l}\text { Van } \\
\text { Oosterwijck } \\
\text { et al. }{ }^{27}\end{array}$ & Fibromyalgia & $\begin{array}{l}\mathrm{n}=30 \\
\mathrm{~F}=26 \\
\text { Age }=45.8 \pm 10.5 \\
\text { years }\end{array}$ & $\begin{array}{l}\text { Neuroscience-ba- } \\
\text { sed pain education (2 } \\
\text { sessions; } 30 \text { minutes) } \\
(n=15)\end{array}$ & $\begin{array}{l}\text { Self-management } \\
\text { (self-management } \\
\text { techniques and han- } \\
\text { dling of daily acti- } \\
\text { vities in relation to } \\
\text { their symptoms). } \\
\text { ( } 2 \text { sessions, } 30 \text { mi- } \\
\text { nutes) ( } n=15)\end{array}$ & 3 months & $\begin{array}{l}\text { FIQ), SF-36, PCI, } \\
\text { PCS, TSK, PVAQ. }\end{array}$ \\
\hline
\end{tabular}

VAS = visual analog scale; PSC = Pain Catastrophizing Scale; WOMAC = Arthritis Self-Efficacy Scale; TSK = Tampa Scale of Kinesiophobia; NDI = Neck Disability Index; GPE = Global Perceived Effect; SF-12v2 = Short Form 12v2 Health Survey; BDI - Beck Depression Inventory; PBCL = Pain Behavior Checklist; CSQR = Coping Strategies Questionnaire Revised; BPI = Brief Pain Inventory; ODQ = Oswestry Disability Questionnaire; DASS = Depression Anxiety Stress Scale; PGIC = Patient Global Impression of Change; $\mathrm{CPCl}=$ Chronic Pain Coping Inventory; PSEQ = Pain Self-Efficacy Questionnaire); FABQ = Fear-Avoidance Beliefs Questionnaire; PBQ = Pain Biology Questionnaire; NRS = Numerical Rating Scale; PSFS = Patient-Specific Functional Scale; SF-36 = Medical Outcomes Study Short Form-36; PRI = Pain Rating Index; SF-MPQ = Short Form-McGill Pain Questionnaire; SF-BDI = Short Version - Beck Depression Inventory; SOPA-D = Survey of Pain Attitudes); NPT = Neurophysiology of Pain Test; PCI = Pain Coping Inventory; RMDQ = Roland Morris Disability Questionnaire; SOPA-R = Survey of Pain Attitudes-Revised; mRMDQ = Roland Morris Disability Questionnaire-Modified; DASS-21 = Depression Anxiety Stress Scales; PRSS = Pain Response Self-Statements Scale; BDI-II = Beck Depression Inventory-II; RMDS = Roland-Morris Disability Scale; CES-D = Center for Epidemiological Studies Depression Scale; QOLS = Quality of Life Scale -QOLS; FIQ = Fibromyalgia Impact Questionnaire; SF-36 = Medical Outcomes Short Form 36 Health Status Survey; PVAQ = Pain Vigilance and Awareness Questionnaire. 
Methodological quality of the studies included

The risk of bias of the articles included in the present systematic review was independently assessed by two reviewers who used the PEDro scale to analyze the methodological quality (Table 2).

\section{Change in pain and disability}

Regarding the primary outcomes (pain intensity and disability), six studies reported pain reduction of at least $10 \%$ and two studies achieved at least 30\% improvement in disability compared to baseline. The percentages of modification for pain and disability, considering the follow-up time are presented in table 3 .

The content of interventions and domains found in the studies Considering the contents covered in the studies of the systematic review it was possible to observe that the most frequent contents included negative thoughts and behavior $(n=5)^{15,19,21,23,29}$, stress management and relaxation techniques $(n=5)^{15,19,21,23,29}$, pain neurophysiology $(n=4)^{16,17,19,23}$, exercises and return to activity $(\mathrm{n}=4)^{15,16,19,29}$ and coping strategies $(\mathrm{n}=3)^{15,23,29}$. Figure 2 shows

Table 2. Methodological quality of the studies included considering the PEDro scale criteria

\begin{tabular}{|c|c|c|c|c|c|c|c|c|c|c|c|c|}
\hline \multirow[t]{2}{*}{ Studies } & \multicolumn{11}{|c|}{ Criteria } & \multirow[t]{2}{*}{ Total } \\
\hline & 1 & 2 & 3 & 4 & 5 & 6 & 7 & 8 & 9 & 10 & 11 & \\
\hline Andersen et al. ${ }^{28}$ & $x$ & $\mathrm{~V}$ & $x$ & $\mathrm{~V}$ & $x$ & $x$ & V & V & V & V & V & 7 \\
\hline Brage et. $\mathrm{al}^{24}$ & V & V & V & V & $x$ & $x$ & V & $x$ & V & $\mathrm{V}$ & V & 7 \\
\hline Carmody et. al..$^{23}$ & V & V & $x$ & V & $x$ & $x$ & V & $x$ & V & V & V & 6 \\
\hline Lefort et al. ${ }^{20}$ & $\mathrm{~V}$ & V & V & $\mathrm{V}$ & $x$ & $x$ & V & V & V & V & V & 8 \\
\hline Meeus et al. ${ }^{26}$ & V & V & V & V & $x$ & $x$ & V & V & $x$ & V & V & 7 \\
\hline Moseley, Nicholas and Hodges ${ }^{18}$ & $\mathrm{~V}$ & $\mathrm{~V}$ & $x$ & $\mathrm{~V}$ & $x$ & $x$ & V & $\mathrm{V}$ & $x$ & V & $\mathrm{V}$ & 7 \\
\hline Nicholas et al. ${ }^{19}$ & V & V & $x$ & $\mathrm{~V}$ & $x$ & $x$ & V & $x$ & V & V & V & 6 \\
\hline Thorn et al. ${ }^{21}$ & $\mathrm{~V}$ & V & $\mathrm{V}$ & $\mathrm{V}$ & $x$ & $x$ & $x$ & $x$ & V & V & V & 6 \\
\hline Van Oosterwijck et al. ${ }^{27}$ & $\mathrm{~V}$ & V & $x$ & $\mathrm{~V}$ & V & $x$ & V & $\mathrm{V}$ & V & V & $\mathrm{V}$ & 8 \\
\hline
\end{tabular}

1 = Eligibility Criteria; 2 = Random allocation; $3=$ Secret allocation; $4=$ Comparability at baseline; 5 = Blinding of participants; $6=$ Blinding of therapists; $7=$ Blinding of evaluators; 8 = Suitable follow-up; 9 = Analysis by intention-to-treat; $10=$ Comparison between groups; $11=$ Measurement of accuracy and variability. $X=$ absence; $\mathrm{V}=$ presence.

Table 3. Percentage of change from baseline on pain and disability outcomes considering the follow-up periods of the studies included

\begin{tabular}{|c|c|c|c|c|c|c|}
\hline \multirow[t]{3}{*}{ Authors } & \multicolumn{6}{|c|}{ Change from baseline (\%) } \\
\hline & \multicolumn{2}{|c|}{ Short ( $\leq 3$ months) } & \multicolumn{2}{|c|}{ Medium (3-6 months) } & \multicolumn{2}{|c|}{ Long (>6 months) } \\
\hline & Pain & Disability & Pain & Disability & Pain & Disability \\
\hline Bennell et al. ${ }^{29}$ & -17.4 & 15.0 & - & - & -24.1 & 20.9 \\
\hline Brage et. $\mathrm{al}^{24}$ & - & - & - & - & 2.0 & 12.2 \\
\hline Chiauzzi et al. ${ }^{15}$ & -7.8 & 6.7 & -9.5 & 5.1 & -14.8 & 2.5 \\
\hline Lefort et al. ${ }^{20}$ & -16.1 & 8.7 & - & - & - & - \\
\hline Moseley, Nicholas, Hodges ${ }^{18}$ & - & 6.6 & - & - & - & - \\
\hline Nicholas et al. ${ }^{19}$ & -12.3 & 23.8 & -12.5 & 20.4 & -9.5 & 16.2 \\
\hline Ris et al. ${ }^{25}$ & - & 2.2 & - & - & - & - \\
\hline
\end{tabular}

The figures in bold meet the inclusion criteria of at least $10 \%$ pain reduction and $30 \%$ improvement in function. 
the frequency of the intervention themes. For the development of the cards of the board domains, the content addressed in at least $50 \%$ of the studies were selected.

\section{Development of the ConbeceDor game}

For the development of the game ConheceDor game, we considered some examples of board games on the market that have a diversity of models, colors, shapes, and objectives. The game consists of a board, 110 cards, one die and the number of markers (pawns) according to the number of participants. The board layout was rectangular measuring $250 \mathrm{~mm}$ by $500 \mathrm{~mm}$, with 50 houses, consisting of a path signaled by the words "Start" and "End," shortcuts, symbols and drawings (Figure 3).

The visual identity of the game ConheceDor is mainly composed of the colors green, yellow and red to attract the eyes to the board. The character present in the identity represents a young man with pain positioned on the spot that the game begins. Along the way, there are several aspects related to pain such as sleep, negative thoughts, drug use, and exercises until the end of the course where the character is illustrating his recovery. The presence of these graphic elements suggests that the theme of the game exemplifies a person with pain until the recovery.

The number of game houses available on the market that were evaluated during the ConheceDor game development goes from 40 to 60. Therefore, the 50 houses for the ConheceDor game were divided into colors (blue, orange, green, red and black), houses "choose your way" and "X" houses. The colors of the houses that represent the game domains were defined by consensus among the developers: negative thoughts (kinesiophobia and catastrophization) (orange), pain neurophysiology (green), stress man- agement (black) relaxation/coping (blue) and physical exercises/ activities (red). The game cards were divided into domain cards according to the colors of the board houses and "X" cards. For the house "choose your way," the player can choose any of the cards of the five domains to respond. The game cards assess the pain knowledge based on "true or false" answers where the player will judge whether a particular statement is beneficial or harmful to a person with pain. The statements used in the game cards were developed based on the scales and questionnaires used to assess patients with pain such as the Tampa Scale of kinesiophobia (TSK), Pain Catastrophizing Scale, Self-efficacy Scale, the Neurophysiological Pain Questionnaire and Attitudes to Pain. When the scales used questions, they have been converted into statements. In house $\mathrm{X}$ the player is punished that can be to stay one round without playing or to return some houses according to the result obtained when throwing the dice. All cards are rectangular with a dimension of $90 \mathrm{~mm} \times 60 \mathrm{~mm}$, printed on millboard, totaling 110 cards being 20 cards per domain and 10 $\mathrm{X}$ cards (Figure 3).

In the beginning, players (maximum of 4) are invited to set the order of the match by throwing the dice. The participant who obtains the highest number will be the first to start the match, followed by the participant with the second highest result, and so on. When moving his marker, the player must follow the command according to the house of the board that can be one of the domain cards, "choose your way" and house "X." The other player will read the card and indicate the correct answer after the first player has answered. If the player's answer is correct, he will have the right to throw the dice once more. If the answer is wrong, he will switch to the other player. It is only allowed to throw the

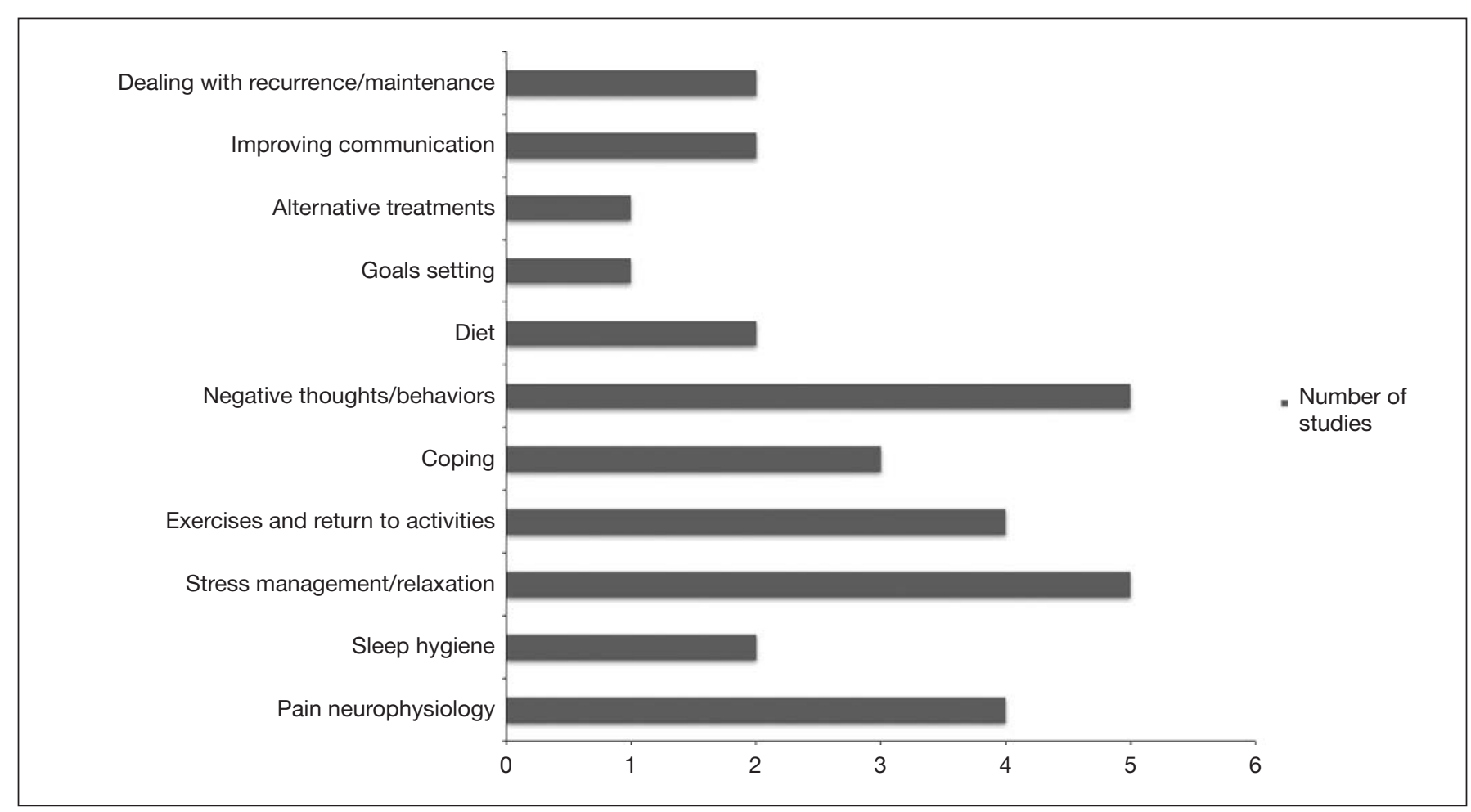

Figure 2. Absolute frequency of the themes in the interventions considering the studies included in the systematic review 


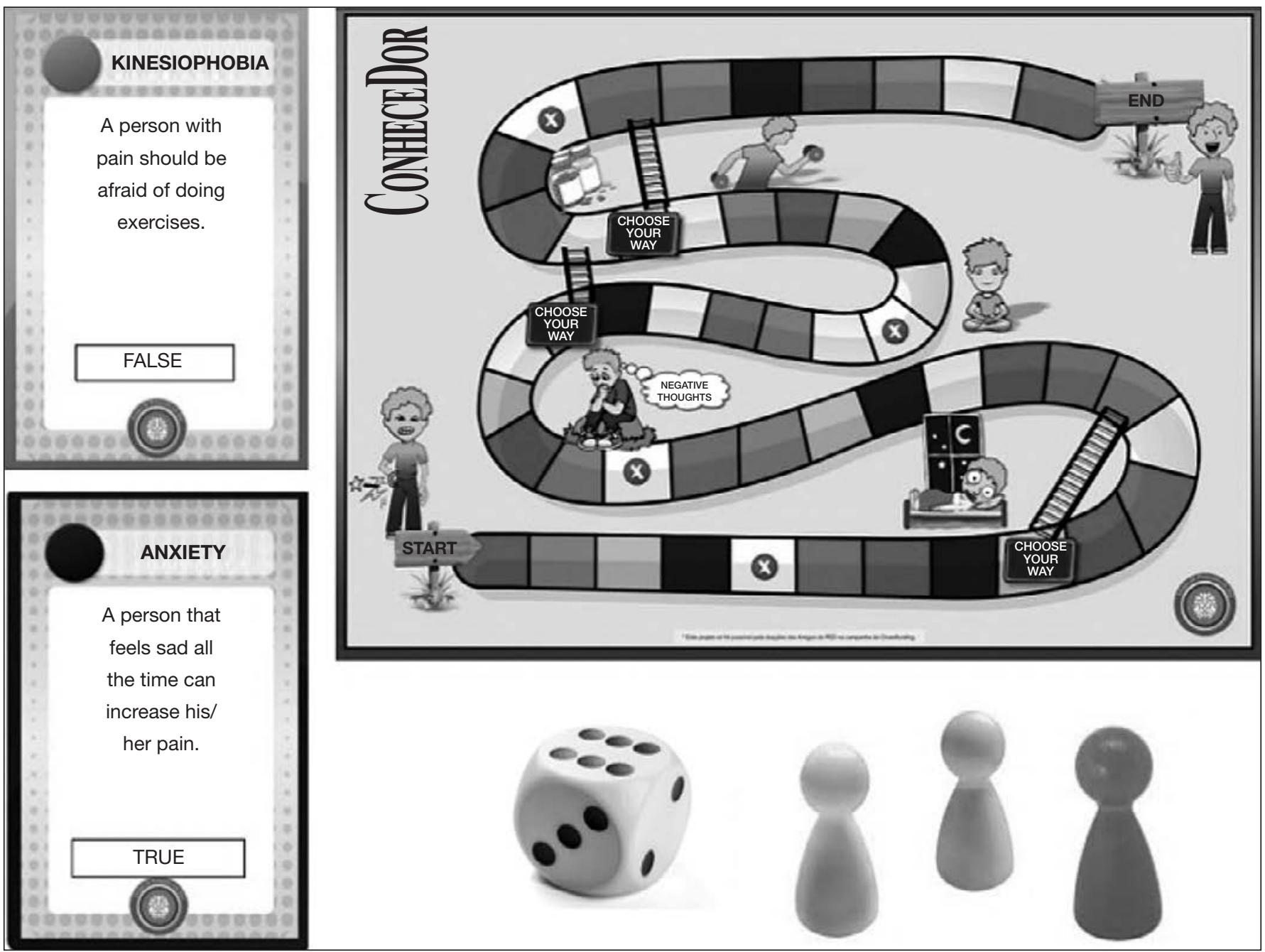

Figure 3. Components of the ConheceDor game (board, cards, dice, and pins)

dice twice, even if the player's answer is correct. In the "choose your way" house, the player can choose the card he wants to answer, and if the answer is correct, he will have the right to follow the "shortcut," shortening his path. The winner will be the one who arrives first at the "FIM" (End) house.

\section{DISCUSSION}

The systematic review of the clinical trials conducted in the present study identified the contents used in PE interventions based on neuroscience and behavioral strategies that contributed to the improvement of pain and disability in people with musculoskeletal pain. In the analysis of the 15 studies that met the inclusion criteria, it was observed that the main contents included the handling of negative thoughts and behavior, pain neurophysiology, stress management and relaxation techniques, coping strategies, exercises and return to activities. A study carried out in Salvador developed a PE booklet addressing the following topics: pain definition, pain classification (acute and chronic), living with pain, myths about pain, strategies for dealing with pain ${ }^{30}$.
The reduction of pain and disability was assessed in the short term (up to three months) by most studies, three studies evaluated in the medium term (three to six months) and five studies in the long term (longer than six months). The reduction in pain intensity ranged from 6.7 to $51.3 \%$ and the improvement in disability from 2.2 to $60 \%$. It is possible that this difference among the studies was due to the characteristics of the population (for example, the health condition addressed), the content of the intervention and the form of administration.

The present study did not seek to investigate the effectiveness of the neuroscience-based PE since other studies have already presented these results ${ }^{11,31,32}$. In the meta-analysis conducted by Geneen et al..$^{32}$, which included different types of educational interventions, the authors did not identify the effect of education on pain intensity relative to the comparison group immediately after and within three months of follow-up. However, for disability, when using neuroscience-based PE, there was evidence of significant improvement immediately after. This effect was not observed in the other types of education investigated in the studies. Other findings in the review by Geneen et al. ${ }^{32}$ included significant improvement in catastrophizing and pain 
awareness only in the studies that used neuroscience-based PE. The systematic review by Louw et al. ${ }^{11}$ showed that neuroscience-based PE improved the knowledge about pain, disability, catastrophizing, pain-related fear, attitudes and behaviors related to pain, return to activities, and decreased the use of Health Services. However, the heterogeneity of the studies was not considered, and the meta-analysis was not performed. Recently, in a systematic review with meta-analysis, it was identified the evidence of moderate quality that the addition of PE to physiotherapy promoted short-term improvement in pain and also moderate evidence for the improvement of disability when PE was conducted isolated or combined with physiotherapy. For the kinesiophobia and catastrophizing outcomes, the authors found no statistical or clinical difference ${ }^{31}$.

The objective of the elaboration of the board game was to develop an educational tool with an interactive interface, facilitating the understanding of theoretical contents about pain and behavioral strategies. Games are considered an active and useful tool in the teaching-learning process of patients. This is only possible because the playfulness allows the acquisition of the concepts attractively and pleasantly. Another characteristic of games is the horizontal relationship between the educator and the learner since games stimulate the interaction among participants, as well as motivating and supporting learning ${ }^{33}$. However, it should be noted that it is still necessary to validate the game with the target audience. During the validation process, it is essential to introduce the game to the target audience so they can evaluate the layout of the game that includes the shape of the board, the colors of the houses, the cards (both their format and the clarity of the information) and the playability that relates to how enjoyable the game is, the time it takes to play and the clarity of the rules. In this way, it is possible that the game needs some modifications after being submitted to this validation process.

Although the development and use of board games in the health area are not very frequent, the few that exist have shown positive results about the learning and education of patients ${ }^{33}$. There are some examples of games in the literature, such as a board game aimed at promoting active and healthy aging. This game acted like a playful pedagogical resource in nursing care, contributing to the construction of knowledge in the elderly health area ${ }^{34}$. Fernandes et al. ${ }^{35}$ described the development of a board game called "Family Nursing Game" aimed at nurses. The participants emphasized the preference for the game, due to the source of interaction and reflection it allows among the participants and for having motivated family care. Pires and Guilhem $^{36}$, developed a board game titled “(IN) DICA-SUS” and realized that the learning sought by the paths of the game for health professionals contemplates the plural aspects of human formation, such as group interaction, active participation, the capacity for self-reflection, the motivation for the study and the willingness to achievement.

It is possible that the intervention strategies using board games have the potential to improve the knowledge about the theoretical content and to facilitate the acquisition or modification of behaviors. During the game, the relationship established be- tween the game and the knowledge is comprehensive due to the numerous cognitive and social phenomena that the game allows the player to experience such as problem-solving, language learning, role-playing, among others ${ }^{37}$.

Although some PE interventions have already been developed for Brazil ${ }^{30,38}$, it is believed that this is the first board game based on a systematic review and critical analysis of the studies on the subject. Also, the characterization of these interventions allowed the development of a board game with previously used content that has demonstrated its effects, especially for pain and disability in people with musculoskeletal pain, based on studies with good methodological quality (PEDro $\geq 6$ ). Thus, this study can contribute by filling a gap in the literature that is the development of educational strategies easy to apply to people with pain. However, this study is not free of limitations either. The biggest was not having conducted the content validation process by a panel of experts and a sample of the target audience. This is a step that should occur after the development of the game, and then the effectiveness of the intervention should be compared to other forms of educational strategies. Therefore, new studies are necessary to evaluate the acceptability, usability, and applicability of the board game by patients and health professionals. These subsequent studies can significantly contribute to fix any issues and to enhance to the board game.

\section{CONCLUSION}

The systematic review followed by the evaluation of the content of the educational interventions allowed to identify the main themes used in clinical trials. This process contributed to the development of a board game for PE that may be a tool to be applied in the clinical practice to treat people with musculoskeletal pain.

Annex 1. Search strategy

\#1 MeSH descriptor: [Chronic Pain] explode all trees

\#2 MeSH descriptor: [Pain] explode all trees

\#3 Widespread Chronic Pain

\#4 MeSH descriptor: [Patient Education as Topic] explode all trees

\#5 Patient Education or Education of Patients

\#6 Education or neuroscience or neurobiology or neurophysiology or pain education or pain science or modern pain education or therapeutic neuroscience education

\#7 MeSH descriptor: [Behavior Therapy] explode all trees

\#8 Randomised controlled trial or clinical trial as a topic or randomised or placebo or randomly or trial

\#9 \#1 or \#2 or \#3

$\# 10$ \#4 or \#5 or \#6 or \#7

$\# 11 \# 8$ and \#9 and \#10

\section{REFERENCES}

1. Global, regional, and national incidence, prevalence, and years lived with disability fo 328 diseases and injuries for 195 countries, 1990-2016: a systematic analysis for the Global Burden of Disease Study 2016. Lancet. 2017;390(10100):1211-59.

2. Simons LE, Elman I, Borsook D. Psychological processing in chronic pain: a neura systems approach. Neurosci Biobehav Rev. 2014;39:61-78.

3. Bushnell MC, Ceko M, Low LA. Cognitive and emotional control of pain and its 
disruption in chronic pain. Nat Rev Neurosci. 2013;14(7):502-11.

4. Crofford LJ. Psychological aspects of chronic musculoskeletal pain. Best Pract Res Clin Rheumatol. 2015;29(1):147-55.

5. Flor H, Turk DC. Chronic pain: an integrated biobehavioral approach. Lippincott Williams \& Wilkins; 2015.

6. Okifuji A, Turk DC. Behavioral and cognitive-behavioral approaches to treating patients with chronic pain: thinking outside the pill box. J Ration Cogn Ther. 2015;33(3):218-38

7. Severeijns R, Vlaeyen JW, van den Hout MA, Weber WE. Pain catastrophizing predicts pain intensity, disability, and psychological distress independent of the level of physical impairment. Clin J Pain. 2001;17(2):165-72.

8. Sullivan MJ, Rodgers WM, Kirsch I. Catastrophizing, depression and expectancies for pain and emotional distress. Pain. 2001;91(1-2):147-54

9. Brox JI, Storheim K, Grotle M, Tveito TH, Indahl A, Eriksen HR. Systematic review of back schools, brief education, and fear-avoidance training for chronic low back pain. Spine J. 2008;8(6):948-58.

10. Engers A, Jellema P, Wensing M, van der Windt DA, Grol R, van Tulder MW. Individual patient education for low back pain. Cochrane Database Syst Rev. 2008;(1):CD004057.

11. Louw A, Zimney K, Puentedura EJ, Diener I. The efficacy of pain neuroscience education on musculoskeletal pain: a systematic review of the literature. Physiother Theory Pract. 2016;32(5):332-55.

12. Nascimento SS. Efeitos neurofisiológicos de terapias cognitivas no manejo da dor: revisão sistemática. Tese de Pós-Graduaçâa em Ciências da Saúde. 2018;

13. Vygotsky LS. A formação social da mente. São Paulo: Martins Fontes; 1984.

14. Nicoletti AA, Filho R. Aprender brincando: a utlizaçáo de jogos, brinquedos e brincadeiras como recursos pedagógicos. Rev Divulg Técnico-Científica ICPG. 2004;2(5):91-4.

15. Chiauzzi E, Pujol LA, Wood M, Bond K, Black R, Yiu E, et al. painACTION-back pain: a self-management website for people with chronic back pain. Pain Med. 2010;11(7):1044-58.

16. Rizzo RR, Medeiros FC, Pires LG, Pimenta RM, McAuley JH, Jensen MP, et al. Hypnosis enhances the effects of pain education in patients with chronic non-specific low back pain: a randomized controlled trial. J Pain. 2018;19(10):1103.e101103-9.

17. Ryan CG, Gray HG, Newton M, Granat MH. Pain biology education and exercise classes compared to pain biology education alone for individuals with chronic low back pain: a pilot randomised controlled trial. Man Ther. 2010;15(4):382-7.

18. Moseley GL, Nicholas MK, Hodges PW. A randomized controlled trial of intensive neurophysiology education in chronic low back pain. Clin J Pain. 2004;20(5):324-30.

19. Nicholas MK, Asghari A, Blyth FM, Wood BM, Murray R, McCabe R, et al. Long-term outcomes from training in self-management of chronic pain in an elderly population: A randomized controlled trial. Pain. 2017;158(1):86-95.

20. LeFort SM, Gray-Donald K, Rowat KM, Jeans ME. Randomized controlled trial of a community-based psychoeducation program for the self-management of chronic pain. Pain. 1998;74(2-3):297-306.

21. Thorn BE, Day MA, Burns J, Kuhajda MC, Gaskins SW, Sweeney K, et al. Randomized trial of group cognitive behavioral therapy compared with a pain education control for low-literacy rural people with chronic pain. Pain. 2011;152(12):2710-20.

22. Gallagher L, McAuley J, Moseley GL. A randomized-controlled trial of using a book of metaphors to reconceptualize pain and decrease catastrophizing in people with chronic pain. Clin J Pain. 2013;29(1):20-5.

23. Carmody TP, Duncan CL, Huggins J, Solkowitz SN, Lee SK, Reyes N, et al. Telephone-delivered cognitive-behavioral therapy for pain management among older military veterans: a randomized trial. Psychol Serv. 2013;10(3):265-75.

24. Brage K, Ris I, Falla D, Søgaard K, Juul-Kristensen B. Pain education combined with neck-and aerobic training is more effective at relieving chronic neck pain than pain education alone-A preliminary randomized controlled trial. Man Ther. 2015;20(5):686-93.

25. Ris I, Søgaard K, Gram B, Agerbo K, Boyle E, Juul-Kristensen B. Does a combination of physical training, specific exercises and pain education improve health-related quality of life in patients with chronic neck pain? A randomised control trial with a 4-month follow up. Man Ther. 2016;26:132-40.

26. Meeus M, Nijs J, Van Oosterwijck J, Van Alsenoy V, Truijen S. Pain physiology education improves pain beliefs in patients with chronic fatigue syndrome compared with pacing and self-management education: a double-blind randomized controlled trial. Arch Phys Med Rehabil. 2010;91(8):1153-9.

27. Van Oosterwijck J, Meeus M, Paul L, De Schryver M, Pascal A, Lambrecht L, et al. Pain physiology education improves health status and endogenous pain inhibition in fibromyalgia: a double-blind randomized controlled trial. Clin J Pain. 2013;29(10):873-82

28. Andersen LN, Juul-Kristensen B, Sørensen TL, Herborg LG, Roessler KK, Søgaard K. Efficacy of tailored physical activity or chronic pain self-management programme on return to work for sick-listed citizens: a 3-month randomised controlled trial. Scand J Public Health. 2015;43(7):694-703.

29. Bennell KL, Nelligan R, Dobson F, Rini C, Keefe F, Kasza J, et al. Effectiveness of an internet-delivered exercise and pain-coping skills training intervention for persons with chronic knee pain: A randomized trial. Ann Intern Med. 2017;166(7):453-62.

30. Mendez SP, Sá KN, Araújo PC, Oliveira IA, Gosling AP, Baptista AF. Elaboration of a booklet for individuals with chronic pain. Rev Dor. 2017;18(3):199-211.

31. Wood L, Hendrick PA. A systematic review and meta-analysis of pain neuroscience education for chronic low back pain: short-and long-term outcomes of pain and disability. Eur J Pain. 2018; [Epub ahead of print]

32. Geneen LJ, Martin DJ, Adams N, Clarke C, Dunbar M, Jones D, et al. Effects of education to facilitate knowledge about chronic pain for adults: a systematic review with meta-analysis. Syst Rev. 2015;4:132.

33. Beinner MA, Morais EA, Reis IA, Reis EA, Oliveira SR. O uso de jogo de tabuleiro na educação em saúde sobre dengue em escola pública. J Nurs UFPE online. 2015;9(4):7304-13.

34. Olympio PC, Alvim NA. Board games: gerotechnology in nursing care practice. Rev Bras Enferm. 2018;71(Suppl 2):818-26. English, Portuguese.

35. Fernandes CS, Martins MM, Gomes BP, Gomes JA, Gonçalves LH. Family nursing game: desenvolvendo um jogo de tabuleiro sobre família. Esc Anna Nery. 2016;20(1):33-7.

36. Pires MR, Guilhem D. Jogo (IN) DICA-SUS: estratégia lúdica na aprendizagem sobre o Sistema Único de Saúde. Texto Context Enferm. 2013;22(2):379-88.

37. Aizencang N. Jugar, aprender y enseñar. Relac que potencian los aprendizajes. 2005;

38. Reis FJ, Bengaly AG, Valentim JC, Santos LC, Martins EF, O'Keeffe M, et al. An E-Pain intervention to spread modern pain education in Brazil. Braz J Phys Ther. 2017;21(5):305-6. 